\title{
Learning specialist skills for a generalist discipline
}

In the European Definition of General Practice, WONCA Europe defined the specialty of General Practice in great detail $^{1}$ but the strength of the definition was actually its generality. Included in this was the statement that GPs provide 'care to every individual seeking medical care irrespective of age, sex and illness.' This splendidly defines the scope of general practice as all encompassing. Implicit also in this is the challenge to our GP specialty registrars of trying to learn the breadth of medicine to the level of an independent practitioner and to apply it regardless of patient or presentation.

In addition, for all GPs there is the task of keeping up to date across the same breadth of medicine. This might seem an impossible challenge but the reality is much more manageable. The expertise ${ }^{2}$ of a GP lies not in being some HAL-like computer (the omniscient computer in the film '2001: A Space Odyssey'). Knowledge will always be important but will increasingly reside outside our heads. Our expertise is in knowing when, where, and how to look. Crucially, GPs' knowledge is demonstrated in interpretation and in making connections between people, problems, and pixels.

Despite this challenge to cover the breadth of medicine through our training and continuing professional development, there seems to be a cacophony of criticism of GPs for not having greater expertise in specific areas.

The Postgraduate Medical Education and Training Board (PMETB) has defined what it requires of approved curricula. These need to be a statement of the intended aims and objectives, content, experiences, outcomes, and processes of an educational programme including: a description of training structure and a description of expected methods of learning, teaching, feedback, and supervision. ${ }^{3}$ The Royal College of General Practitioners (RCGP) fulfils all that the PMETB requires of it. The GP curriculum (www.rcgp-curriculum.org.uk) is a commendable tome but it runs the risk of overloading both trainees and educators.

In this issue of the BJGP, Burke and colleagues outline an inclusive approach to developing a genetics curriculum. ${ }^{4}$ Undoubtedly, the rapid pace of developments in genetics will lead to an increasing role in medicine. ${ }^{5}$ As the human genome is better understood we will better recognise the genetic components of disease and, based on this, the propensity to develop disease. It is likely that our consultations will move in a short period from the current practice of discussing the family history of a few serious conditions and referring to a specialist genetics service, to dealing with ideas and concerns arising from positive genetic tests that may have dire personal consequences.

We may question the ethics of these developments, but advances in genetics will alter society's view of what is acceptable. The Roslin Institute sheep, Dolly, would no longer shock or perhaps even be challenged. In addition, the days of genetic manipulation are perhaps not far off, with one man's Frankenstein scenario being another's lifeline. Burke et al acknowledge the demands placed on those learning for the specialty of general practice ('specialty learning time'); therefore, her team has focused on achieving consensus about what they feel must be included. Their approach has led to a sensible statement within the RCGP curriculum in that pressure to include greater detail about genetics has been balanced with that from the many competing areas.

Contrasting medical training with other disciplines, such as nursing, we see the medical model providing craft knowledge and skills, but it is for the practitioner to apply them. Other disciplines focus on specific skills, which leads to tight boundaries of practice. The application of knowledge is key to the role of GP. Thus the communication expected of a GP about genetics is the application of our general communication learning; genetic risk discussions are similar to the discussions we have about the risk of cardiovascular diseases.

Like the UK, Australia and Denmark have moved towards competence-based curricula. These too are based on general principles but with supporting statements. At McMaster University the Canadians have stuck much more to principles stating that: the physician is an effective clinician, family medicine is community based, the family physician is a resource to a defined practice population, and that the physician-patient relationship is central to family medicine. ${ }^{6}$ Focusing on general principles, as do all four of these curricula, emphasises the scope of general practice. It is these principles which define our being, and which we pass on to future generations.

The role of the GP is constantly changing. As a pragmatic profession we accommodate that which comes our way. The Quality and Outcomes Framework was rapidly assimilated into our working practices and each iteration of the framework is similarly accommodated. Our role will continue to change. With the advent of 'Modernising Scientific Careers' and 'Modernising Nursing Careers' following on closely from the NHS's 'Modernising Medical Careers', it is likely that there will be new healthcare scientist and nursing roles. Already we have nurseled practices and the role of specialist nurses in areas such as diabetes and respiratory disease has enabled GPs to focus on other areas such as the management of complex multisystem disease. As a discipline we need to be prepared for our new role alongside these colleagues.

Surgeons and physicians have become much more specialised; indeed, the general surgeon of our youth is now a rarity. While there is a case for GPs with a special interest, this is additional to the role of a GP. The strength of our discipline 
is our generalism. The management of complex multisystem disease in the patients' context is a specialist skill, one of the specialist skills of our generalist discipline.

As medical knowledge expands and the delivery of care shifts closer to the patient there will be pressures on the knowledge of GPs and increasing expectations placed upon them. A reductionist approach risks de-professionalisation and lends itself to greater regulation without recognising the value and adaptability of our generalist freedoms.

Returning to the RCGP curriculum as an indicator of what is expected of our discipline, the core statement, Being a General Practitioner, ${ }^{7}$ is the curriculum and the following statements are interpretations of topic areas to be seen through the lens of the core statement. For example, a 'holistic approach' will manifest differently in a young man with an earache compared to a mother with terminal ovarian cancer. Illustrations illuminate our understanding of what the core statement means by 'holism'.

The GP curriculum is the educational statement of what it is to be a generalist: a 'general practitioner'. Already it appears that some trainers and registrars are treating the curriculum as a topic tick-box exercise as they are focusing on the supporting statements to the detriment of the whole. The craft and skills of general practice will be lost among the chaff. The core statement, Being a General Practitioner, as the curriculum for general practice describes what most of us recognise as a GP and is sufficient now and for the future. This should be our guide.

The RCGP Curriculum (http://www.rcgpcurriculum.org.uk) was developed to PMETB specifications. It is a splendid work and was commended as such by the PMETB when approved by them as the curriculum for GP training. It is already undergoing a robust programme of development. Its apparent vastness runs the risk of being its downfall, but only if we let it be so. If we remember its purpose and use it as our guide, but recognise that the specialist skills for a generalist discipline are unique and do not lend themselves to a reductionist approach, then we should and can focus on preparing tomorrow's GPs as described in Being a General Practitioner.

\section{Simon Gregory}

General Practice Postgraduate Dean, East

Midlands Healthcare Workforce Deanery,

Leicester \& Nottingham

\section{REFERENCES}

1. WONCA Europe. The European definition of general practice/family medicine. London: WONCA Europe, 2005.

2. Dreyfus HL, Dreyfus SE. Mind over machine: the power of human intuition and expertise in the era of the computer. Free Press: Michigan. 1986.

3. Postgraduate Medical Education and Training Board. Standards for curricula and assessment systems. London: Postgraduate Medical Education and Training Board, 2008.

4. Burke S, Martyn M, Stone A, et al. Developing a curriculum statement based on clinical practice: genetics in primary care. Br J Gen Pract 2009; 59: 99-103.

5. Royal College of General Practitioners. Genetics in primary care. North West England Faculty of the Royal College of General Practitioners. A report from the Faculty Genetics Group. Blackpool, April 1995. Occas Pap R Coll Gen Pract 1998; (77): 1-12.

6. McMaster University. Postgraduate family medicine residency program family medicine objectives. http://fammedmcmaster.ca/postgrad/handbook/goalsand-objectives/Family\%20Medicine\%20Objectives.pdf (accessed 13 Dec 2008).

7. Royal College of General Practitioners. Curriculum for specialty training for general practice. the core statement Being a General Practitioner. London: RCGP, 2007.

DOI: 10.3399/bjgp09X395058

\section{ADDRESS FOR CORRESPONDENCE}

\section{Simon Gregory}

East Midlands Healthcare Workforce

Deanery, Rutland House, 11 Merus Court,

Meridian Way, Leicester, LE19 1RJ.

E-mail: Simon.Gregory@eastmidlands.nhs.uk 